\title{
The Strong Sequential Core for Two-period Economies
}

Citation for published version (APA):

Predtetchinski, A., Herings, P. J. J., \& Peters, H. J. M. (2002). The Strong Sequential Core for Two-period Economies. Journal of Mathematical Economics, 38, 465-482. https://doi.org/10.1016/S03044068(02)00078-2

Document status and date:

Published: 01/01/2002

DOI:

10.1016/S0304-4068(02)00078-2

Document Version:

Publisher's PDF, also known as Version of record

\section{Please check the document version of this publication:}

- A submitted manuscript is the version of the article upon submission and before peer-review. There can be important differences between the submitted version and the official published version of record.

People interested in the research are advised to contact the author for the final version of the publication, or visit the DOI to the publisher's website.

- The final author version and the galley proof are versions of the publication after peer review.

- The final published version features the final layout of the paper including the volume, issue and page numbers.

Link to publication

\footnotetext{
General rights rights.

- You may freely distribute the URL identifying the publication in the public portal. please follow below link for the End User Agreement:

www.umlib.nl/taverne-license

Take down policy

If you believe that this document breaches copyright please contact us at:

repository@maastrichtuniversity.nl

providing details and we will investigate your claim.
}

Copyright and moral rights for the publications made accessible in the public portal are retained by the authors and/or other copyright owners and it is a condition of accessing publications that users recognise and abide by the legal requirements associated with these

- Users may download and print one copy of any publication from the public portal for the purpose of private study or research.

- You may not further distribute the material or use it for any profit-making activity or commercial gain

If the publication is distributed under the terms of Article $25 \mathrm{fa}$ of the Dutch Copyright Act, indicated by the "Taverne" license above, 


\title{
The strong sequential core for two-period economies
}

\author{
Arkadi Predtetchinski ${ }^{\text {a,* }}$, P. Jean-Jacques Herings ${ }^{\text {a }}$, Hans Peters ${ }^{\text {b, } 1}$ \\ ${ }^{a}$ Department of Economics, Universiteit, Maastricht, P.O. Box 616, 6200 MD Maastricht, The Netherlands \\ ${ }^{\mathrm{b}}$ Department of Quantitative Economics, Universiteit Maastricht, P.O. Box 616, 6200 MD, \\ Maastricht, The Netherlands
}

Received 26 June 2001; received in revised form 3 September 2002; accepted 10 September 2002

\begin{abstract}
The strong sequential core for two-stage economies with a possibly incomplete set of assets in period zero and trade in commodities in period one consists of those goods allocations that are in the classical core and moreover, after realization of the state of nature, in the core of the economy where executed asset contracts serve as initial endowments. The strong sequential core coincides with the classical core when all possible state-contingent contracts may serve as an asset. For finance economies it is shown that the strong sequential core is generically empty when there is an incomplete set of assets. Outside the setting of finance economies, we show that the strong sequential core can be empty even if there is a complete set of assets. If the set of constrained feasible allocations resulting from trading in assets, is enlarged to include also allocations outside the agents' consumption sets, then a complete set of assets is sufficient for the equivalence of the resulting semi-strong sequential core and the classical core.
\end{abstract}

(C) 2002 Elsevier Science B.V. All rights reserved.

Keywords: Core; Assets; Uncertainty

\section{Introduction}

The usual implicit assumption for a cooperative solution concept like the core is that the agents can write binding contracts on outcomes provided by that concept. In a static situation like a one-shot cooperative game or an exchange economy it is not essential whether a core allocation is regarded as a candidate for a binding contract or whether it is directly interpreted as one. Agreeing on such an allocation is equivalent to carrying it out

\footnotetext{
* Corresponding author. Tel.: +31-43-38883635, fax: +31-43-38884878.

E-mail addresses: a.predtetchinski@algec.unimaas.nl (A. Predtetchinski), p.herings@algec.unimaas.nl (P.J.-J. Herings), h.peters@ke.unimaas.nl (H. Peters).

1 Tel.: +31-43-3883288; fax: +31-43-3884874.
} 
since nothing happens in between. This is radically different in a dynamic situation, even in the case of complete certainty. Agents or coalitions might change their minds after an originally agreed upon contract has partially been carried out, because it might no longer be in their best interest to stick to it. In the case of uncertainty, where information becomes available over time, this is even more likely to occur.

We consider the case of a two-period exchange economy under uncertainty. In period zero there is trade in assets, the payoffs of which depend on the future, unknown, state of the world. In period one the uncertainty is resolved, asset contracts are executed and on this basis trade in commodities takes place. In the ex ante or classical core as in Aumann (1961), coalitions consider the allocations that they can achieve in each state of nature by pooling their endowments, and compute their ex ante utilities over these allocations. A classical core allocation consists of a bundle of goods for each agent in each state of nature such that no coalition can improve ex ante. In this notion asset trading does not play a role since in any state of nature any redistribution of the initial endowments is agreed upon ex ante and is compatible with any trade in assets in period zero.

The classical core concept, however, fails to take into account that agents can reconsider their positions in the subeconomy at period one after resolution of the uncertainty. Then asset trading becomes important since it determines the initial positions in each state of nature. Coalitions might be able to improve upon the initial classical core allocation ex post. A classical core allocation might not be incentive compatible once the state of nature in period one is known. Similar point of views have been taken in Gale (1978), Repullo (1988), and Koutsougeras (1998), who discuss sequential core concepts, and Kranich et al. (2001), who study multi-period models under certainty where at each period the agents face a cooperative game or an exchange economy.

To capture the implications of selfenforcement, we impose on top of the conditions of the classical core, the requirement that in each state of nature the resulting allocation is in the core of the subeconomy in which executed asset contracts serve as initial endowments. The set of allocations satisfying these requirements is called the strong sequential core.

The strong sequential core is a very selective solution concept. As is evident from the definition, it is a refinement of the classical core. The strong sequential core is therefore useful to study how robust classical core elements are with respect to new blocking opportunities that arise due to changed circumstances. It also allows us to study how existing assets can be used to limit the creation of new blocking opportunities. Moreover, the strong sequential core is a subset of both the weak sequential core as studied in Predtetchinski et al. (2002) and of the two-stage core as introduced in Koutsougeras (1998). Therefore, when the strong sequential core is non-empty, it gives a sharp and a reliable prediction for the outcome of the cooperation in the two-period economies.

The strong sequential core is weakly increasing in the number of available assets: the more assets there are in the economy, the larger is the set of allocations that are robust to coalitional deviations in period $t=1$, the larger is the strong sequential core. When each possible contingent contract may serve as an asset, so there is an asset for each commodity contingent on each state of nature, the strong sequential core coincides with the classical core. Indeed, it is possible to implement the classical core allocation directly by an appropriate trade in assets at period zero. Retrading at period one cannot lead to improvements by definition of the classical core. 
For the general case with an incomplete set of assets, the strong sequential core can be either empty or non-empty. For the special case of finance economies, i.e., one good is traded at each state of nature, it is possible to obtain the sharper result that the strong sequential core is generically (with respect to the initial endowments) empty if the number of assets is two or more less than the number of states.

An obvious question is whether equivalence with the classical core is still obtained if there is only a complete set of assets in the sense of Arrow (1953). The surprising answer is that not only equivalence may fail to hold, but even that the strong sequential core may be empty. We show that this is partially due to the requirement, implicit in the definition of the strong sequential core, that the endowments in the subeconomies resulting from asset trading in period zero, must be in the consumption sets of the agents.

We also define the so-called semi-strong sequential core. In that definition it is no longer required that the endowments at the beginning of period one belong to the consumption sets of the agents. We show that when there is a complete set of assets, then equivalence of the classical core and the semi-strong sequential core obtains.

Throughout the paper we assume symmetric information. Dynamic aspects of cooperation also play a role in economies with private information, where usually a third stage between the ex ante and ex post stages is distinguished, namely an interim stage in which the agents learn their private information. See for instance Vohra (1999) for a study of the core in this framework. In these models, however, subject to incentive compatibility constraints, a complete contracting environment results; for the special case of symmetric information one is lead to the classical core. The study of asymmetric information issues in an incomplete contracting framework remains an interesting subject for further research.

The organization of this paper is as follows. Section 2 specifies the model and some preliminaries, and Section 3 defines the strong sequential core. Section 4 considers the special case of finance economies and Section 5 treats the general multiple commodity case. Section 6 defines the semi-strong sequential core and states our equivalence result. Section 7 concludes. The more involved proofs are collected in the Appendix A.

\section{The model and preliminaries}

\subsection{The model}

We consider an economy with two time periods $(t=0,1)$ and uncertainty concerning period one. Uncertainty is modelled as a finite set $\{1, \ldots, S\}$ of states of nature with given probabilities $\rho_{s}>0, s=1, \ldots, S$ of occurrence. Period $t=0$ is identified with state $s=0$.

There is a set $N=\{1, \ldots, n\}$ of agents. Agents trade in $J$ assets in period 0 and, conditional on the realization of the state of nature $s$, in $L$ commodities in period 1 . In state of nature $s=1, \ldots, S$, agent $i$ has a consumption set $X_{s}^{i} \subset \mathbb{R}^{L}$, so the consumption set of agent $i$ is given by $X^{i}=\prod_{s=1}^{S} X_{s}^{i}$. We denote $X=\prod_{i \in N} X^{i}$. An agent $i$ is further characterized by his vector of initial endowments in state of nature $s, \omega_{s}^{i} \in X_{s}^{i}$, and his elementary (Bernoulli) utility function $u_{s}^{i}: X_{s}^{i} \rightarrow \mathbb{R}$. Agents are expected utility maximizers, with $v^{i}: X^{i} \rightarrow \mathbb{R}$ the expected utility function defined by $v^{i}\left(x^{i}\right)=\sum_{s=1}^{S} \rho_{s} u_{s}^{i}\left(x_{s}^{i}\right)$. 
The matrix of asset payoffs is given by the $S L \times J$ matrix $A$. The generic entry $A_{s, l}^{j}$ of the matrix $A$ specifies the quantity of commodity $l$ paid by asset $j$ in state of nature $s$.

These components together define our economy, denoted by $\mathcal{E}$. We shall often parametrize economies by the agents' endowments $\omega$. A typical element of this family is denoted by $\mathcal{E}_{\omega}$.

The institutional set-up of the economy is as follows:

1. In period 0 , trade in assets takes place. Alternatively, one may think of these trades taking the form of state-contingent contracts. There are no endowments and therefore no consumption in period $t=0$.

2. Nature randomly chooses the state of nature. The execution of asset contracts takes place and results in an allocation $x$.

3. Trade in commodities takes place. Agents treat allocation $x$ as their initial endowments. Trade in commodities results in an allocation $y$ of commodities, which is consumed.

Notice that the institutional setting is one of dynamic exchange without markets. In particular, no prices are formed. Our analysis is therefore complementary to the extensive literature on constrained suboptimality of competitive equilibria when asset markets are incomplete, which originates from the contribution of Geanakoplos and Polemarchakis (1986).

An important role in our analysis is played by the the set of constrained feasible allocations

$$
\mathcal{A}=\left\{x \in X \mid \sum_{i \in N} x^{i}=\sum_{i \in N} \omega^{i}, x^{i}-\omega^{i} \in\langle A\rangle\right\}
$$

where $\langle A\rangle$ denotes the linear space spanned by the columns of $A$, i.e. the span of $A$. This is the set of allocations that can be achieved by trade in assets in period 0 . The set of allocations that are constrained feasible under the initial endowments $\omega$ will also be denoted by $\mathcal{A}_{\omega}$.

\subsection{Subeconomies}

The dynamic structure of the economy allows for the identification of a number of subeconomies.

The ex ante economy, i.e. the one that precedes the resolution of uncertainty, may be associated with state of nature 0 . This is the economy with commodity space $\mathbb{R}^{S L}$, consumption sets $X^{i}$, expected utility functions $v^{i}$, and initial endowments $\omega^{i}$. It is denoted by $\mathcal{E}_{0}$. Formally

$$
\mathcal{E}_{0}=\mathcal{E}\left(N, \mathbb{R}^{S L},\left\langle X^{i}, v^{i}, \omega^{i}\right\rangle_{i \in N}\right)
$$

The classical core of the economy $\mathcal{E}_{0}$ is denoted by $C\left(\mathcal{E}_{0}\right)$, hence

$$
\begin{aligned}
C\left(\mathcal{E}_{0}\right)= & \left\{x \in X \mid \sum_{i \in N} x^{i}=\sum_{i \in N} \omega^{i} ; \quad \nexists T \subseteq N, \nexists y \in \prod_{i \in T} X^{i}, \quad\right. \text { such that } \\
& \left.\sum_{i \in T} y^{i}=\sum_{i \in T} \omega^{i} \text { and } v^{i}\left(y^{i}\right)>v^{i}\left(x^{i}\right) \text { for all } i \in T\right\}
\end{aligned}
$$


Any constrained feasible allocation $x$ gives rise to exactly $S$ ex post subeconomies, one in each of the possible states of nature, following the resolution of uncertainty. The economy associated with state of nature $s$ has commodity space $\mathbb{R}^{L}$, consumption sets $X_{s}^{i}$, utility functions $u_{s}^{i}$, and initial endowments $x_{s}^{i}$. It is denoted by $\mathcal{E}_{s, x}$. Formally

$$
\mathcal{E}_{s, x}=\mathcal{E}\left(N, \mathbb{R}^{L},\left\langle X_{s}^{i}, u_{s}^{i}, x_{s}^{i}\right\rangle_{i \in N}\right)
$$

The classical core of the economy $\mathcal{E}_{s, x}$ is denoted by $C\left(\mathcal{E}_{s, x}\right)$.

\subsection{Example}

Throughout the paper we exploit the following example. Consider the family of economies, parameterized by the agents' endowments $\omega \in \Omega=\mathbb{R}_{++}^{n S L}$, in which $N=\{1,2\}$ and both agents have identical consumption sets and utility functions given by

$$
\begin{aligned}
& X_{s}^{i}=\mathbb{R}_{++}^{L} \\
& u_{s}^{i}\left(y_{s}^{i}\right)=\sum_{l=1}^{L} \ln \left(y_{s l}^{i}\right), \quad y_{s}^{i} \in \mathbb{R}_{++}^{L} \\
& v^{i}\left(y^{i}\right)=\sum_{s=1}^{S} \frac{1}{S} \sum_{l=1}^{L} \ln \left(y_{s l}^{i}\right), \quad y^{i} \in \mathbb{R}_{++}^{S L}
\end{aligned}
$$

Note the implicit assumption that all states are equally likely. Let the initial endowments $\omega \in \Omega$ and the constrained feasible allocation $x \in \mathcal{A}_{\omega}$ be given. Define the numbers

$$
\begin{aligned}
& \alpha_{s}^{i}(x)=\left(\prod_{l=1}^{L} \frac{x_{s l}^{i}}{x_{s l}^{\Sigma}}\right)^{1 / L} \\
& \alpha_{0}^{i}(x)=\left(\prod_{s=1}^{S} \prod_{l=1}^{L} \frac{x_{s l}^{i}}{x_{s l}^{\Sigma}}\right)^{1 / S L}
\end{aligned}
$$

where $x^{\Sigma}=x^{1}+x^{2}$. Then

$$
\mathcal{P}\left(\mathcal{E}_{0, \omega}\right)=\left\{y \in \mathbb{R}_{++}^{n S L} \mid \exists t^{i}>0, \quad \text { such that } \quad y^{i}=t^{i} \omega^{\Sigma}, \quad t^{1}+t^{2}=1\right\}
$$

is the set of Pareto optimal allocations and

$$
\begin{aligned}
& C\left(\mathcal{E}_{0, \omega}\right)=\left\{y \in \mathbb{R}_{++}^{n S L} \mid \exists t^{i}>0, \quad \text { such that } y^{i}=t^{i} \omega^{\Sigma}, \quad t^{1}+t^{2}=1, \quad t^{i} \geq \alpha_{0}^{i}(\omega)\right\} \\
& C\left(\mathcal{E}_{s, x}\right)=\left\{y_{s} \in \mathbb{R}_{++}^{n L} \mid \exists t^{i}>0, \quad \text { such that } \quad y_{s}^{i}=t^{i} \omega_{s}^{\Sigma}, \quad t^{1}+t^{2}=1, \quad t^{i} \geq \alpha_{s}^{i}(x)\right\}
\end{aligned}
$$


are the classical cores of the subeconomies at states $s=0,1, \ldots, S$. These expressions will be helpful in expressing the the strong (and the semi-strong) sequential core for the economy $\mathcal{E}_{\omega}$.

\section{The strong sequential core}

We start with an example that illuminates some essential points behind the concept of the strong sequential core.

\subsection{Example}

Consider the economy of Section 2.3 with two commodities and two possible states of nature. The two agents have identical consumption sets and utility functions defined in (1)-(3). The initial endowments are

$$
\left(\begin{array}{c}
\omega^{1} \\
\omega^{2}
\end{array}\right)=\left(\begin{array}{cccc}
1-\epsilon & 1-\epsilon & \epsilon & \epsilon \\
\epsilon & \epsilon & 1-\epsilon & 1-\epsilon
\end{array}\right)
$$

where the first two columns correspond to $s=1$ and the last two to $s=2$. Assume for a moment that there are no assets available in the economy. Then agents begin the exchange game in period $t=1$ having their initial endowments $\omega$. Moreover, the initial allocation is the only allocation that is robust to the coalitional deviations ex post, in period one. Hence, it is the only candidate to belong to the strong sequential core. However, the initial allocation is extremely unattractive from the ex ante viewpoint: in fact, for $\epsilon$ close to zero, it is one of the worst outcomes that may be implemented in the economy! It is very unlikely that such an allocation will be agreed upon in period $t=0$ : nearly every feasible allocation would be a profitable deviation from $\left(\omega^{1}, \omega^{2}\right)$. In this situation the requirements of the ex post and the ex ante coalitional stability are incompatible, and the strong sequential core is empty.

An important idea behind the strong sequential core is that if the set of the available assets enlarges, then more allocations become robust to coalitional deviations in period $t=1$.

Suppose that there is an asset in the economy, whose payoffs are given by the vector $A=(1,0,-1,0)$. This asset pays one unit of commodity $l=1$ in state of nature $s=1$ and minus one unit of the same commodity in state of nature $s=2$. It is easy to see that in the presence of such an asset the strong sequential core is nonempty. Indeed, the following exchange may be arranged in period zero: agent 1 gives $1-2 \epsilon$ units of the asset to agent 2. This asset trade would result in an allocation

$$
\left(\begin{array}{l}
\bar{x}^{1} \\
\bar{x}^{2}
\end{array}\right)=\left(\begin{array}{cccc}
\epsilon & 1-\epsilon & 1-\epsilon & \epsilon \\
1-\epsilon & \epsilon & \epsilon & 1-\epsilon
\end{array}\right) \in \mathcal{A}
$$

In the trade that follows the execution of contracts the fully symmetric allocation that assigns one half of each commodity to each agent in each state of nature is robust to coalitional deviations in period $t=1$. Moreover, such an allocation belongs to the classical core in the ex ante subeconomy, and is therefore an element of the strong sequential core. 


\subsection{Definition of the strong sequential core}

Definition 1. An allocation $\bar{y} \in X$ is an element of the strong sequential core of the economy $\mathcal{E}$, denoted by $\operatorname{SSC}(\mathcal{E})$, if

1. $\bar{y} \in C\left(\mathcal{E}_{0}\right)$.

2. There exists $\bar{x} \in \mathcal{A}$ such that $\bar{y}_{s} \in C\left(\mathcal{E}_{s, \bar{x}}\right)$ for all $s=1, \ldots, S$.

Point 1 of the definition requires that there are no deviations from $\bar{y}$ in period $t=0$. Point 2 guarantees that there are no deviations in the subsequent period, given appropriate asset trades arranged at the outset.

In the absence of assets, the only constrained feasible allocation is $\omega$. Consequently, only those allocations are robust to deviations at period $t=1$, which belong to the period one cores $C\left(\mathcal{E}_{s, \omega}\right)$. However, when there are assets in the economy, the grand coalition can support different allocations by redistributing assets among its members in period zero. We may think of the grand coalition as redistributing assets in period $t=0$ of the economy in order to prevent subcoalitions from deviating in the subsequent period. According to this interpretation, agents agree on asset trades and final consumption in period 0: since final consumption is in the ex ante core, they have no reason to deviate before the state of nature realizes. Then condition 2 guarantees that agents do not deviate in period 1 .

Note that the strong sequential core increases when the set of constrained feasible allocations increases. If, in particular, $A$ has rank $S L$, then every classical core allocation can be sustained as an allocation in the strong sequential core. Indeed, any classical core allocation can be implemented directly by an appropriate trade in assets. At the arrival of period 1, contracts are executed, and no retrading of commodities is needed. Summarizing, we get the following theorem.

Theorem 1. If the set of assets traded in an economy expands, the strong sequential core weakly increases. If $A$ has rank $S L$, then $\operatorname{SSC}(\mathcal{E})=C\left(\mathcal{E}_{0}\right)$.

An important case where the rank of $A$ is $S L$ is the one where for each commodity contingent on each state, there is a contract specifying its delivery in period 1 . This corresponds to the complete market structure as analyzed in traditional general equilibrium theory. Nevertheless, the requirement that the rank of $A$ equals $S L$ is very demanding. The next two sections consider the more interesting case where some assets are missing.

\section{Finance economies}

We start out with the special case of finance economies. In a finance economy there is just one commodity in each state of nature. We assume that $X_{s}^{i}=\mathbb{R}_{+}$. If the utility functions are strongly monotone, then for any $x \in \mathcal{A}$ and $s=1, \ldots, S$

$$
C\left(\mathcal{E}_{s, x}\right)=\left\{x_{s}\right\}
$$

This implies that the strong sequential core is given by

$$
\operatorname{SSC}(\mathcal{E})=\mathcal{A} \cap C\left(\mathcal{E}_{0}\right)
$$


We make the following standard assumptions on the utility functions.

\section{Assumption $\mathcal{U}$.}

1. $v^{i}: \mathbb{R}_{+}^{S} \rightarrow \mathbb{R}$ is continuous on $\mathbb{R}_{+}^{S}$ and twice continuously differentiable on $\mathbb{R}_{++}^{S}$.

2. For any $x^{i} \in \mathbb{R}_{++}^{S}, \nabla v^{i}\left(x^{i}\right) \in \mathbb{R}_{++}^{S}$.

3. For any $x^{i} \in \mathbb{R}_{++}^{S},\left\{y^{i} \in \mathbb{R}_{+}^{S} \mid v^{i}\left(y^{i}\right) \geq v^{i}\left(x^{i}\right)\right\} \subseteq \mathbb{R}_{++}^{S}$.

4. Indifference curves have non-zero Gaussian curvature, $\left[x^{i} \in \mathbb{R}_{++}^{S}, h \in \mathbb{R}^{S}, h \neq\right.$ $\left.0, h^{T} \nabla v^{i}\left(x^{i}\right)=0\right]$ implies $\left[h^{T} \nabla^{2} v^{i}\left(x^{i}\right) h<0\right]$.

The proof of the following result is given in the Appendix A.

Theorem 2. Let $\mathcal{E}_{\omega}$ be a family of finance economies parameterized by the agent's initial endowments $\omega \in \Omega=\mathbb{R}_{++}^{S n}$. Suppose that $n \geq 2$, all $v^{i}$ satisfy assumption $\mathcal{U}$, and rank $(A)=J$.

1. If $J<S-1$, then there exists a set of full measure $\Omega^{*} \subset \Omega$ such that for all $\omega \in \Omega^{*}$

$$
\operatorname{SSC}\left(\mathcal{E}_{\omega}\right)=\emptyset
$$

2. If $J=S-1$, then there exists a set of full measure $\Omega^{*} \subset \Omega$ such that for all $\omega \in \Omega^{*}$

$\operatorname{SSC}\left(\mathcal{E}_{\omega}\right)$ is either empty or finite.

Theorem 2 implies that if trade in assets is limited in the sense that the rank of the asset matrix is smaller than $S-1$, then there is a set of initial endowments for which the strong sequential core is generically empty. If the rank of the asset matrix is equal to $S-1$, then there is a set of initial endowments for which the strong sequential core is generically empty or finite. The latter statement cannot be strengthened in either direction. More precisely, when $J=S-1$, then there may exist two complementary subsets $\bar{\Omega}$ and $\underline{\Omega}$ of $\Omega$, both with non-empty interiors, such that for all economies in the set $\bar{\Omega}$ the strong sequential core is non-empty, and for all those in the set $\Omega$ the strong sequential core is empty. This is demonstrated by the following example.

Example 1. Consider the following family of finance economies $\mathcal{E}_{\omega}$ parameterized by the agents' endowments $\omega \in \Omega=\mathbb{R}_{++}^{n S}$. We assume that $S \geq 3, N=\{1,2\}$ and the agents have identical consumption sets and utility functions defined by (1)-(3). The $S \times(S-1)$ matrix of asset payoffs is given by

$$
A=\left(\begin{array}{cccc}
1 & 0 & \cdots & 0 \\
0 & 1 & \cdots & 0 \\
\vdots & \vdots & \ddots & \vdots \\
0 & 0 & \cdots & 1 \\
0 & 0 & \cdots & 0
\end{array}\right)
$$


The set of Pareto-efficient allocations and the core of the economy $\mathcal{E}_{0, \omega}$ are defined in (4) and (7). Then

$$
\operatorname{SSC}\left(\mathcal{E}_{\omega}\right)=C\left(\mathcal{E}_{0, \omega}\right) \cap \mathcal{A} \subseteq \mathcal{P}\left(\mathcal{E}_{0, \omega}\right) \cap \mathcal{A}=\left\{\left(t^{1}(\omega) \omega^{\Sigma}, t^{2}(\omega) \omega^{\Sigma}\right)\right\}
$$

where $t^{i}(\omega)=\omega_{S}^{i} / \omega_{S}^{\Sigma}$, the fraction of the total endowment in state $S$ owned by agent $i$.

The strong sequential core of the economy $\mathcal{E}_{\omega}$ is therefore either an empty set or a single-element set defined by (9). It is a single-element set if the allocation defined by (9) is individually rational, and it is empty otherwise

$$
\operatorname{SSC}\left(\mathcal{E}_{\omega}\right)= \begin{cases}\left\{t^{1}(\omega) \omega^{\Sigma}, t^{2}(\omega) \omega^{\Sigma}\right\} & \text { if } t^{i}(\omega) \geq \alpha_{0}^{i}(\omega) \text { for } i=1,2 \\ \varnothing & \text { otherwise }\end{cases}
$$

Define $\bar{\Omega} \subset \Omega$ to be the set of initial allocations $\omega \in \Omega$ satisfying

$$
t^{i}(\omega) \geq \alpha_{0}^{i}(\omega), \quad i=1,2
$$

and let $\underline{\Omega}$ be its complement in $\Omega$.

It remains to show that both sets have non-empty interiors. Whenever $S \geq 3$, an allocation

$$
\left(\begin{array}{l}
\bar{\omega}^{1} \\
\bar{\omega}^{2}
\end{array}\right)=\left(\begin{array}{llllll}
S & 1 & 1 & \ldots & 1 & 1 \\
1 & S & 1 & \ldots & 1 & 1
\end{array}\right)
$$

satisfies condition (8) with strict inequalities for both agents 1 and 2 . Hence, it lies in the interior of $\bar{\Omega}$. In contrast, allocation

$$
\left(\begin{array}{c}
\underline{\omega}^{1} \\
\underline{\omega}^{2}
\end{array}\right)=\left(\begin{array}{cccccc}
S & S & S & \ldots & S & 1 \\
1 & 1 & 1 & \ldots & 1 & S
\end{array}\right)
$$

is such that $t^{1}(\underline{\omega})<\alpha_{0}^{1}(\underline{\omega})$. Hence, it is an interior point of the set $\underline{\Omega}$.

\section{The multiple commodities case}

Let the number of commodities be arbitrary, $L \geq 1$. The first observation is that Theorem 2 cannot be extended to economies with multiple goods. When $L>1$, then, irrespective of the number of assets, there exist robust examples of economies with a non-empty strong sequential core. That is, there is a subset $\bar{\Omega} \subseteq \Omega$ with non-empty interior, such that for all economies in $\bar{\Omega}$ the strong sequential core is non-empty. Such an example is the following one.

Example 2 (Robust existence of the strong sequential core). We consider the family of economies $\mathcal{E}_{\omega}$ parameterized by the agents' endowments $\omega \in \Omega=\mathbb{R}_{++}^{n S L}$. We assume that $L \geq 2, N=\{1,2\}$ and that the agents have identical consumption sets and utility functions defined by (1)-(3). Assume that there are no assets. 
The cores for the economies in $\mathcal{E}_{0, \omega}$ and $\mathcal{E}_{s, \omega}$ are given by (7) and (8). The strong sequential core of the economy $\mathcal{E}_{\omega}$ without assets is

$$
\begin{aligned}
\operatorname{SSC}\left(\mathcal{E}_{\omega}\right)=\{ & \left\{y \in \mathbb{R}_{++}^{n S L} \mid \exists t^{i}>0, \quad\right. \text { such that } \\
& \left.y^{i}=t^{i} \omega^{\Sigma}, \quad t^{1}+t^{2}=1, t^{i} \geq \alpha_{0}^{i}(\omega) \quad t^{i} \geq \alpha_{s}^{i}(\omega), \quad s=1, \ldots, S\right\}
\end{aligned}
$$

It is non-empty if and only if the following condition is satisfied. There are no $s, s^{\prime} \in$ $\{0,1, \ldots, S\}, s \neq s^{\prime}$, such that

$$
\alpha_{s}^{1}(\omega)+\alpha_{s^{\prime}}^{2}(\omega)>1
$$

If this condition is not satisfied, then the requirement of individual rationality for agent 1 in state of nature $s$ is not compatible with the one for agent 2 in the state $s^{\prime}$. Let $\bar{\Omega}$ be the set of initial allocations $\omega \in \Omega$ satisfying condition (9). The allocation

$$
\left(\begin{array}{c}
\bar{\omega}_{s}^{1} \\
\bar{\omega}_{s}^{2}
\end{array}\right)=\left(\begin{array}{ccccc}
1 & \epsilon & 1 & \ldots & 1 \\
\epsilon & 1 & 1 & \ldots & 1
\end{array}\right), \quad s \in\{1, \ldots, S\}
$$

for $\epsilon<1$, is such that for all $s, s^{\prime} \in\{0,1, \ldots, S\}, s \neq s^{\prime}$, the strict inequalities

$$
\alpha_{s}^{1}(\omega)+\alpha_{s^{\prime}}^{2}(\omega)<1
$$

hold true. Therefore, this allocation is an interior point of the set $\bar{\Omega}$.

We conclude that for all economies in the interior of the set $\bar{\Omega}$ the strong sequential core is non-empty. Due to Theorem 1, we can augment the economy by any number of assets while preserving the robust nonemptiness of the strong sequential core.

It has already been noted (Theorem 1) that the strong sequential core weakly increases in the rank of matrix $A$ and that it coincides with $C\left(\mathcal{E}_{0}\right)$ when the rank of $A$ reaches $S L$. In the remainder of this section we explore the case of a strongly complete set of assets, by which me mean the following.

Definition 2. For any vector $p \in \mathbb{R}^{S L}$, let $p \cdot A$ be the $S \times J$-dimensional matrix with rows $p_{s} A_{s}, s=1, \ldots, S$. There is a strongly complete set of assets if for every $p \in \mathbb{R}_{++}^{S L}$ $\operatorname{rank}(p \cdot A)=S$

In particular, this 'condition' implies that the rank of matrix $A$ is not less than $S$. Under this assumption of strong completeness, financial markets equilibria exist, coincide with equilibria in the complete markets model, and are therefore Pareto-efficient, see Arrow (1953).

There are at least two reasons why one might conjecture that under strong completeness the strong sequential core is non-empty, and in fact equal to the classical core. The first intuition is based on the case of finance economies, where strong completeness is equivalent to the requirement that the rank of $A$ be $S$. We have already shown that in the setting of finance economies the strong sequential core is equal to classical core when this rank condition holds. The second intuition comes indeed from the above mentioned equivalence between the complete markets model and an economy as described in Arrow (1953) with a 
sequential structure, but a complete set of Arrow securities. Note also that Theorem 1 states equivalence to the classical core when the rank of $A$ equals $L S$.

It turns out, however, that such a conjecture is false. Not only is there no equivalence to the classical core. The strong sequential core might even be empty when $L \geq 2$ and the set of assets is strongly complete. A reason for the emptiness of the strong sequential core is found in the definition of $\mathcal{A}$, the set of constrained feasible allocations. The requirement that a constrained feasible allocation $x$ should lie in a consumption set may prevent the strong sequential core from existence, even when there is a strongly complete set of assets. This is illustrated in the following example, and elaborated in the next section, where we consider the semi-strong sequential core.

Example 3 (Non-existence for a strongly complete set of assets). Consider the family of economies parameterized by the agents' endowments $\omega \in \Omega=\mathbb{R}_{++}^{n S L}$, in which $N=\{1,2\}$ and the consumption sets and utility functions are as in (1)-(3). We specify only the last $L+1$ rows of the asset matrix $A$

$$
\begin{aligned}
& A_{S-1, L}=\{0, \ldots, 0,1\} \\
& A_{S, l}=\{0, \ldots, 0,0\}, \text { for } l=1, \ldots, L-1 \\
& A_{S, L}=\{0, \ldots, 0,1\}
\end{aligned}
$$

The other entries of the matrix may be chosen arbitrarily. This implies that the rank of $A$ does not exceed $S L-L$. Note, however, that the case of a strongly complete set of assets is not excluded. For instance, if $S=3$ and $L=2$ the matrix

$$
A=\left(\begin{array}{llll}
1 & 0 & 0 & 0 \\
0 & 1 & 0 & 0 \\
0 & 0 & 1 & 0 \\
0 & 0 & 0 & 1 \\
0 & 0 & 0 & 0 \\
0 & 0 & 0 & 1
\end{array}\right)
$$

has the required structure and satisfies the criterion in Definition 2.

We claim that all economies in some open subset of $\Omega$ have an empty strong sequential core.

The classical cores of the economies $\mathcal{E}_{0, \omega}$ and $\mathcal{E}_{s, x}$ are given by (7) and (8). The strong sequential core of the economy $\mathcal{E}_{\omega}$ is the set

$$
\begin{aligned}
& \operatorname{SSC}\left(\mathcal{E}_{\omega}\right)=\left\{y \in \mathbb{R}_{++}^{n S L} \mid \exists t^{i}>0, \quad \text { such that } \quad y^{i}=t^{i} \omega^{\Sigma}, t^{1}+t^{2}=1, t^{i} \geq \alpha_{0}^{i}(\omega)\right. \\
& \left.\exists x \in \mathcal{A}_{\omega}, \quad \text { such that } t^{i} \geq \alpha_{s}^{i}(x), s=1, \ldots, S\right\}
\end{aligned}
$$

Take any $\omega \in \Omega$ that satisfies the following inequalities

$$
\omega_{S, L}^{1}-\omega_{S-1, L}^{1}>0 \quad\left(\frac{\omega_{S, L}^{1}-\omega_{S-1, L}^{1}}{\omega_{S, L}^{1}}\right)^{1 / L} \alpha_{S}^{1}(\omega)+\alpha_{0}^{2}(\omega)>1
$$


and any $x \in \mathcal{A}_{\omega}$. There exists a scalar $\theta$ (agent 1 's net trade in asset $J$ ) such that

$$
\begin{aligned}
& x_{S-1, L}^{1}=\omega_{S-1, L}^{1}+\theta \\
& x_{S, l}^{1}=\omega_{S, l}^{1}, \quad l=1, \ldots, L-1 \\
& x_{S, L}^{1}=\omega_{S, L}^{1}+\theta
\end{aligned}
$$

From the positivity constraint $x_{S-1, L}>0$ it follows that $\theta>-\omega_{S-1, L}^{1}$ and that $x_{S, L}>$ $\omega_{S, L}^{1}-\omega_{S-1, L}^{1}$. Therefore

$$
\alpha_{S}^{1}(x)=\alpha_{S}^{1}(\omega)\left(\frac{x_{S, L}^{1}}{\omega_{S, L}^{1}}\right)^{1 / L} \geq \alpha_{S}^{1}(\omega)\left(\frac{\omega_{S, L}^{1}-\omega_{S-1, L}^{1}}{\omega_{S, L}^{1}}\right)^{1 / L}
$$

which implies

$$
\alpha_{S}^{1}(x)+\alpha_{0}^{2}(\omega)>1
$$

The last inequality says that the condition of individual rationality for agent 1 in state of nature $S$ is not compatible with the condition of individual rationality for agent 2 in state of nature 0 . Because $x$ was chosen arbitrarily in $\mathcal{A}_{\omega}$, this implies that the strong sequential core is empty.

A transfer of commodities from agent 1 to agent 2 in state of nature $S$ might solve the problem. It could diminish the value of $\alpha_{S}^{1}(\cdot)$ and thus weaken the condition of individual rationality for agent 1 in state of nature $S$. However, the only asset that pays in state of nature $S$ is asset $J$ and it also pays in state of nature $S-1$. Its payoffs are denominated in the units of commodity $L$. Given that the initial endowment $\omega_{S-1, L}^{1}$ is sufficiently small, any attempt to redistribute a unit of asset $J$ from agent 1 to agent 2 results in an allocation $x$ that prescribes to agent 1 a negative amount $x_{S-1, L}^{1}$ of commodity $L$ in state of nature $S-1$. However, such an allocation is prohibited by the definition of $\mathcal{A}$.

Thus, even though there is a strongly complete set of assets, there is no way to redistribute commodities that become available to agent 1 in state of nature $S$. Any allocation $x$ that may potentially arise as a result of such a redistribution will be outside the consumption set, and therefore is ruled out by the definition of $\mathcal{A}$. This discussion leads to a natural weakening of the strong sequential core, discussed in the next section.

\section{The semi-strong sequential core}

We first modify the definition of the set of constrained feasible allocations.

Definition 3. Let

$$
\mathcal{A}^{\prime}=\left\{x \in \mathbb{R}^{n S L} \mid \sum_{i \in N} x^{i}=\sum_{i \in N} \omega^{i}, x^{i}-\omega^{i} \in\langle A\rangle\right\}
$$

be the set of semi-constrained feasible allocations. An allocation $\bar{y} \in X$ is an element of the semi-strong sequential core, denoted by $\operatorname{SSC}^{\prime}(\mathcal{E})$, if 
1. $\bar{y} \in C\left(\mathcal{E}_{0}\right)$.

2. there exists an $\bar{x} \in \mathcal{A}^{\prime}$ such that $\bar{y}_{s} \in C\left(\mathcal{E}_{s, \bar{x}}\right), s=1, \ldots, S$.

The set of semi-constrained feasible allocations contains all allocations $x$ that may potentially arise as a result of trade in assets, even though some of the commodity bundles specified by these allocations may lie outside the consumption set. The semi-strong sequential core allows for the interpretation that agents may have debts at the beginning of period 1, that is an allocation outside the consumption set. By the end of period 1 all debts must be paid back, so that the final allocation $\bar{y}$ belongs to the consumption set.

Definition 3 involves an extension of the classical core to those economies $\mathcal{E}_{s, x}$, whose initial endowments $x$ do not belong to the consumption sets. To illustrate some implications of this, define the set of allocations feasible for a coalition $M \subseteq N$ in state $s$, given the initial allocation $x \in \mathcal{A}^{\prime}$, as

$$
F_{s, x}(M)=\left\{y_{s} \in \prod_{i \in M} \mathbb{R}_{+}^{L} \mid \sum_{i \in M} y_{s}^{i}=\sum_{i \in M} x_{s}^{i}\right\}
$$

Then the classical core of the economy $\mathcal{E}_{s, x}$ is the set of all allocations $\bar{y}_{s} \in F_{s, x}(N)$ such that no $M \subseteq N$ and $y_{s} \in F_{s, x}(M)$ exist with $u_{s}^{i}\left(y_{s}^{i}\right)>u_{s}^{i}\left(\bar{y}_{s}^{i}\right)$ for all $i \in M$. If the aggregate endowment $\sum_{i \in M} x_{s}^{i}$ is inconsistent with individual consumption bundles in the respective consumption sets of the agents of a coalition $M$, then the feasibility set for $M$ is empty. Coalition $M$ can improve upon no allocation in the state of nature $s$. A coalition can deviate only if it is able to pay back the aggregate debt of all its members. In particular, the conditions of individual rationality are valid only for those agents whose initial endowments are non-negative. It should be stressed that the extension of the classical core to a wider class of economies does not involve the extension of the utility functions beyond the consumption sets.

The main result of this section is the following theorem, which states the equivalence of the classical core and the strong sequential core when there is a strongly complete set of assets. Its proof is in the Appendix A.

Theorem 3. Suppose that the economy $\mathcal{E}$ satisfies the following assumptions: the consumption sets $X^{i}$ are convex; the utility functions $u_{s}^{i}$ are continuous, concave, and strongly monotone; $\left\{y^{i} \in X^{i} \mid v^{i}\left(y^{i}\right) \geq v^{i}\left(\omega^{i}\right)\right\} \subseteq$ int $\left(X^{i}\right)$; and there is a strongly complete set of assets. Then $\operatorname{SSC}^{\prime}(\mathcal{E})=C\left(\mathcal{E}_{0}\right)$.

The following example shows that an economy with an incomplete set of assets may have an empty semi-strong sequential core.

Example 4. Consider the family of economies parameterized by the agents' endowments $\omega \in \Omega=\mathbb{R}_{++}^{n S L}$, in which $N=\{1,2\}$. As before, the consumption sets and utility functions are given by (1)-(3). We assume $A_{S}=0$, so the case with a strongly complete set of assets is excluded. Other entries of the matrix $A$ may be chosen arbitrarily. Then for all economies in some open subset of $\Omega$ the semi-strong sequential core is empty. 
Notice that

$$
\begin{aligned}
C\left(\mathcal{E}_{s, x}\right)= & \left\{y_{s} \in \mathbb{R}_{++}^{n L} \mid \exists t^{i}>0, \quad\right. \text { such that } \\
& \left.y_{s}^{i}=t^{i} \omega_{s}^{\Sigma}, t^{1}+t^{2}=1, t^{i} \geq \alpha_{s}^{i}\left(x_{s}\right), \quad \text { whenever } \quad x_{s}^{i} \in \mathbb{R}_{++}^{L}\right\}
\end{aligned}
$$

and

$$
\begin{aligned}
\operatorname{SSC}^{\prime}\left(\mathcal{E}_{\omega}\right)= & \left\{y \in \mathbb{R}_{++}^{n S L} \mid \exists t^{i}>0, \exists x \in \mathcal{A}_{\omega}^{\prime}, \quad\right. \text { such that } \\
& y^{i}=t^{i} \omega^{\Sigma}, t^{1}+t^{2}=1, t^{i} \geq \alpha_{0}^{i}(\omega) \quad t^{i} \geq \alpha_{s}^{i}\left(x_{s}\right), \\
& \text { whenever } \left.\quad x_{s}^{i} \in \mathbb{R}_{++}^{L}\right\}
\end{aligned}
$$

Note that the conditions of individual rationality are only valid for those agents $i$ whose bundle $x_{s}^{i}$ is strictly positive.

The requirement $A_{S}=0$ implies that $x_{S}=\omega_{S}$ for any semi-constrained feasible allocation $x$. Therefore, for any $\omega$ satisfying

$$
\alpha_{S}^{i}\left(\omega_{S}\right)+\alpha_{0}^{j}(\omega)>1(i \neq j)
$$

the semi-strong sequential core of the economy $\mathcal{E}_{\omega}$ is empty.

The last inequality implies that the condition of individual rationality for agent $i$ in state of nature $S$ is not compatible with the condition of individual rationality for agent $j$ in state of nature 0 . Redistribution of commodities from agent $i$ to agent $j$ in state $S$ of the world could diminish the value of $\alpha_{S}^{i}(\cdot)$ and thus weaken the condition of individual rationality for agent $i$ in state $S$. However, redistribution of goods that become available in state $S$ via trade in assets is not possible. In this way incompleteness of the set of assets may result in the emptiness of the semi-strong sequential core.

\section{Concluding remarks}

The fact that most economic interaction takes place over time has received very limited attention in the part of the economic literature that focuses on cooperative solution concepts like the core. In this paper, we study the implications arising from the unraveling of time and uncertainty for the concept of the core. The strong sequential core imposes an additional requirement of time consistency on the classical core, in the sense that a strong sequential core allocation can be implemented without any coalition having an incentive to deviate at any point in time.

The strong sequential core highlights a stabilizing property of assets. The strong sequential core is weakly increasing in the number of assets traded. The strong sequential core is shown to be equivalent to the classical core when each possible contingent contract can be traded before the resolution of uncertainty. Surprisingly, equivalence and even non-emptiness of the strong sequential core may fail under quite stringent notions of completeness of the set of assets being traded.

The possible emptiness of the strong sequential core suggests to consider weakenings of it. One is to allow for debts at the beginning of period one, leading to the semi-strong 
sequential core, which is also studied in this paper. The semi-strong sequential core leads to equivalence to the classical core when the set of assets traded is strongly complete, but might still be empty otherwise. Another weakening of the strong sequential core can be obtained by requiring that possible blocking allocations of coalitions are credible in the sense that they should belong to the strong sequential core of the economy restricted to that coalition. This weak sequential core concept is studied in Predtetchinski et al. (2002). Although the weak sequential core is a superset of the strong sequential core, it might still be empty when the set of assets is not sufficiently complete. Blending time and uncertainty with cooperative solution concepts therefore points at serious problems in the implementation of such concepts, in particular when it is only possible to trade in a limited set of contracts at the outset.

\section{Acknowledgements}

Helpful comments of Andres Perea and of the participants of the European Workshop on General Equilibrium Theory, May 2001, Maastricht, are gratefully acknowledged.

\section{Appendix A. Proofs}

In this Appendix A we provide proofs of Theorems 2 and 3.

\section{A.1. Proof of Theorem 2}

We start with a lemma.

Lemma 1. Consider a finance economy $\mathcal{E}$, in which the consumption sets $X^{i}$ are closed and bounded from below, and the Bernoulli utility functions $u_{s}^{i}$ are continuous and strongly monotone. Then the strong sequential core of the economy $\mathcal{E}$ is a compact set.

Proof. Recall that the strong sequential core of $\mathcal{E}$ is given by

$$
\operatorname{SSC}(\mathcal{E})=C\left(\mathcal{E}_{0}\right) \cap \mathcal{A}
$$

Under the assumption that the sets $X^{i}$ are bounded from below, the set $\mathcal{A}$ of constrained feasible allocations for the economy $\mathcal{E}$ is bounded. It remains to be verified that the strong sequential core is a closed set.

Consider the sequence $y_{q}$ in $\operatorname{SSC}(\mathcal{E})$ converging to a point $y_{0} \in \mathbb{R}^{S n}$. Then, since the sets $X^{i}$ are closed, $y_{0} \in \mathcal{A}$. Suppose that there is a coalition $M \subseteq N$ and an allocation $\tilde{y}$, feasible for $M$, such that $v^{i}\left(\tilde{y}^{i}\right)>v^{i}\left(y_{0}^{i}\right)$ for all $i \in M$. Then, due to the continuity of $v^{i}$, for $q$ large enough the inequalities $v^{i}\left(\tilde{y}^{i}\right)>v^{i}\left(y_{q}^{i}\right)$ for all $i \in M$ hold true, implying that $y_{q}$ is not an element of $C\left(\mathcal{E}_{0}\right)$, contradicting the definition of $y_{q}$. Consequently, $y_{0} \in C\left(\mathcal{E}_{0}\right)$.

We use the following additional definitions. 
- $\Xi=\left\{(\omega, \theta) \in \Omega \times \mathbb{R}^{J n} \mid \omega^{i}+A \theta^{i} \in \mathbb{R}_{++}^{S}, \sum_{i=1}^{n} \theta^{i}=0\right\}$, a smooth $(S n+J n-J)$ dimensional manifold.

- $\mathcal{S}=\left\{z \in \mathbb{R}_{++}^{S} \mid\|z\|=1\right\}$, the intersection of the strictly positive orthant with the unit sphere, a smooth $(S-1)$-dimensional manifold.

- $\mathcal{S}^{n}$, the product of $n$ unit spheres $\mathcal{S}$, a smooth $n(S-1)$-dimensional manifold.

- $\Delta=\left\{\left(z^{1}, \ldots, z^{n}\right) \in \mathcal{S}^{n} \mid z^{i}=z^{j}, i, j=1, \ldots, n\right\}$, a smooth $(S-1)$-dimensional submanifold of $\mathcal{S}^{n}$.

- $g^{i}: \mathbb{R}_{++}^{S} \rightarrow \mathcal{S}$, a normalized gradient of the function $v^{i}$, defined as

$$
g^{i}\left(y^{i}\right)=\frac{\nabla v^{i}\left(y^{i}\right)}{\left\|\nabla v^{i}\left(y^{i}\right)\right\|}, \forall y^{i} \in \mathbb{R}_{++}^{S}
$$

- $h: \Xi \rightarrow \mathcal{S}^{n}$, a function defined by

$$
h(\omega, \theta)=\left(g^{1}\left(\omega^{1}+A \theta^{1}\right), \ldots, g^{n}\left(\omega^{n}+A \theta^{n}\right)\right), \forall(\omega, \theta) \in \Xi
$$

For a smooth manifold $M$, and $\bar{x}$ a point in $M, T_{\bar{x}}(M)$ denotes the tangent space to $M$ at $\bar{x}$. We claim that the linear mapping

$$
D_{\xi} h(\bar{\xi}): T_{\bar{\xi}}(\Xi) \rightarrow T_{h(\bar{\xi})}\left(\mathcal{S}^{n}\right)
$$

is surjective for any $\bar{\xi} \in \Xi$. To show this we only need the fact that the linear mappings $D_{x^{i}} g^{i}\left(\bar{x}^{i}\right): \mathbb{R}^{S} \rightarrow T_{g^{i}\left(\bar{x}^{i}\right)}(\mathcal{S})$ are surjective, which follows immediately from the non-zero Gaussian curvature of $v^{i}$, see Debreu (1972).

Let $\bar{\xi}=(\bar{\omega}, \bar{\theta}), \bar{x}^{i}=\bar{\omega}^{i}+A \bar{\theta}^{i}, i=1, \ldots, n$. Take any vectors $d g^{i} \in T_{g^{i}\left(\bar{x}^{i}\right)}(\mathcal{S})$, so that $d g \in T_{h(\bar{\xi})}\left(\mathcal{S}^{n}\right)$. The surjectiveness of $D_{x^{i}} g^{i}\left(\bar{x}^{i}\right)$ implies that there are vectors $d \omega^{i} \in \mathbb{R}^{S}$ such that

$$
D_{x^{i}} g^{i}\left(\bar{x}^{i}\right) d \omega^{i}=d g^{i}
$$

If we set $d \theta^{i}$ all equal to zero, then $d \xi=(d \omega, d \theta) \in T_{\bar{\xi}}(\Xi)$, and

$$
D_{\xi} h(\bar{\xi}) d \xi=d g
$$

Since $d g$ was chosen arbitrarily in the tangent space of $\mathcal{S}^{n}$, this shows the surjectivity of the $D_{\xi} h(\bar{\xi})$.

Surjectivity of the differential $D_{\xi} h(\bar{\xi})$ for all $\bar{\xi} \in \Xi$ implies that the function $h$ is transverse to any submanifold of $\mathcal{S}^{n}$. In particular, $h$ is transverse to $\Delta$. The preimage of $\Delta$ under $h$

$$
\begin{aligned}
h^{-1}(\Delta)= & \left\{(\omega, \theta) \in \Omega \times \mathbb{R}^{J n} \mid \omega^{i}+A \theta^{i} \in \mathbb{R}_{++}^{S}, \quad \sum_{i=1}^{n} \theta^{i}=0,\right. \\
& \left.g^{i}\left(\omega^{i}+A \theta^{i}\right)=g^{k}\left(\omega^{k}+A \theta^{k}\right) i, k=1, \ldots, n\right\}
\end{aligned}
$$

is non-empty. Moreover, it is a smooth submanifold of $\Xi$, and its dimension is given by

$$
\begin{aligned}
\operatorname{dim} h^{-1}(\Delta) & =\operatorname{dim} \Xi-\operatorname{dim} \mathcal{S}^{n}+\operatorname{dim} \Delta \\
& =(S n+J n-J)-n(S-1)+(S-1)=(n-1)(J+1)+S
\end{aligned}
$$


Let $p r: h^{-1}(\Delta) \rightarrow \Omega$ be defined by $\operatorname{pr}(\omega, \theta)=\omega$ for all $(\omega, \theta) \in \Xi$. If $J<S-1$, then $\operatorname{dim} h^{-1}(\Delta)<\operatorname{dim} \Omega$. By Sard's Theorem the projection of $h^{-1}(\Delta)$ into $\Omega$ has Lebesgue measure zero. Therefore, there is a set of full measure $\Omega^{*} \subset \Omega$ such that $\operatorname{pr}^{-1}(\omega)=\emptyset$ for all $\omega \in \Omega^{*}$. If $J=S-1$, then $\operatorname{dim} h^{-1}(\Delta)=\operatorname{dim} \Omega$. In this case, there is a set of full measure $\Omega^{*} \subset \Omega$ such that for all $\omega \in \Omega^{*}$ the set $\operatorname{pr}^{-1}(\omega)$ is a zero-dimensional manifold. Observe that any manifold of dimension zero is a discrete set.

Under the assumptions of the theorem, the strong sequential core of the finance economy $\mathcal{E}_{\omega}$ is contained in the set

$$
\left\{x \in \mathbb{R}_{++}^{S n} \mid \sum_{i=1}^{n} x^{i}=\sum_{i=1}^{n} \omega^{i}, x^{i}-\omega^{i} \in\langle A\rangle, \quad g^{i}\left(x^{i}\right)=g^{k}\left(x^{k}\right), i, k=1, \ldots, n\right\}
$$

which is homeomorphic to $\mathrm{pr}^{-1}(\omega)$. Moreover, $\operatorname{SSC}\left(\mathcal{E}_{\omega}\right)$ is a compact set. The observation that any compact and discrete set is finite completes the proof.

\section{A.2. Proof of Theorem 3}

Let $\mathcal{P}\left(\mathcal{E}_{0}\right)$ and $\mathcal{P}\left(\mathcal{E}_{s, \omega}\right)$ be the sets of Pareto-efficient allocations for the economies $\mathcal{E}_{0}$ and $\mathcal{E}_{s, \omega}$, respectively. Theorem 3 is proved in three steps

1. $\left[y \in \mathcal{P}\left(\mathcal{E}_{0}\right)\right]$ implies $\left[y_{s} \in \mathcal{P}\left(\mathcal{E}_{s, \omega}\right)\right.$ for all $\left.s \in\{1, \ldots, S\}\right]$.

2. $\left[y_{s} \in \mathcal{P}\left(\mathcal{E}_{s, \omega}\right) \cap \operatorname{int}\left(X_{S}\right)\right.$ for all $\left.s \in\{1, \ldots, S\}\right]$ implies $\left[\exists x \in \mathcal{A}^{\prime}: y_{s} \in C\left(\mathcal{E}_{s, x}\right)\right.$ for all $s \in\{1, \ldots, S\}]$.

3. $\operatorname{SSC}(\mathcal{E})=C\left(\mathcal{E}_{0}\right)$.

Step 1. Suppose that there is an allocation $y$ which is Pareto-efficient in the economy $\mathcal{E}_{0}$ and not Pareto-efficient in the economy $\mathcal{E}_{\sigma, \omega}$ in some state $\sigma \in\{1, \ldots, S\}$. Then there exists an allocation $\tilde{y}_{\sigma} \in X_{\sigma}$, such that

$$
\sum_{i \in N} \tilde{y}_{\sigma}^{i}=\sum_{i \in N} \omega_{\sigma}^{i} ; \quad u_{\sigma}^{i}\left(\tilde{y}_{\sigma}^{i}\right) \geq u_{\sigma}^{i}\left(y_{\sigma}^{i}\right) \forall i \in N
$$

with some strict inequality. Define the allocation $\hat{y}$ as follows:

$$
\hat{y}_{s}^{i}= \begin{cases}\tilde{y}_{\sigma}^{i} & \text { if } s=\sigma \\ y_{s}^{i} & \text { otherwise. }\end{cases}
$$

Then allocation $\hat{y}$ is feasible in the economy $\mathcal{E}_{0}$, and

$$
v^{i}\left(\hat{y}^{i}\right) \geq v^{i}\left(y^{i}\right) \forall i \in N
$$

with some strict inequality, contradicting the fact that $y$ is Pareto-efficient in the state zero economy.

Step 2. Take an allocation $y$ such that $y_{s} \in \mathcal{P}\left(\mathcal{E}_{s, \omega}\right) \cap \operatorname{int}\left(X_{S}\right)$ for all $s \in\{1, \ldots, S\}$. Under the assumptions of the theorem, the Second Welfare theorem implies that there exist vectors of commodity prices $p_{s} \in \mathbb{R}_{++}^{L}$ such that $\left(y_{s}, p_{s}\right)$ is an equilibrium with transfers of the 
economy $\mathcal{E}_{s, \omega}$. Let the distribution of wealth in this equilibrium be given by $\left(w_{s}^{1}, \ldots, w_{s}^{n}\right)$. By the assumption of a complete set of assets, the system of equations

$$
\left(\begin{array}{c}
w_{1}^{i} \\
\vdots \\
w_{S}^{i}
\end{array}\right)=\left(\begin{array}{c}
p_{1} \omega_{1}^{i} \\
\vdots \\
p_{S} \omega_{S}^{i}
\end{array}\right)+\left(\begin{array}{c}
p_{1} A_{1} \\
\vdots \\
p_{S} A_{S}
\end{array}\right) \theta^{i}
$$

has a solution with respect to $\theta^{i}$ for all $i \in N$. Denote this solution by $\bar{\theta}^{i}$ and let $x^{i}=\omega^{i}+A \bar{\theta}^{i}$. It is obvious that $x \in \mathcal{A}^{\prime}$.

Since $p_{s} x_{s}^{i}=w_{s}^{i}$ for all $i \in N,\left(y_{s}, p_{s}\right)$ is a Walrasian equilibrium of the economy $\mathcal{E}_{s, x}$ (of the state $s$ economy with initial allocation $x$ ). The observation that any equilibrium allocation of the economy $\mathcal{E}_{s, x}$ is an element of the classical core of this economy completes Step 2.

Step 3. Take allocation $y$ in $C\left(\mathcal{E}_{0}\right)$, arbitrarily. Then the conditions of individual rationality, $v^{i}\left(y^{i}\right) \geq v^{i}\left(\omega^{i}\right)$, imply that $y^{i} \in \operatorname{int}\left(X^{i}\right)$. Moreover, $y$ is Pareto-optimal in the economy $\mathcal{E}_{0}$. Steps 1 and 2 then imply that $y$ is an element of the semi-strong sequential core. This completes the proof since it follows readily from the definition of the strong sequential core that $\operatorname{SSC}(\mathcal{E}) \subset C\left(\mathcal{E}_{0}\right)$.

\section{References}

Arrow, K.J., 1953. Le Rôle des valeurs boursières pour la rèpartition la meilleure des risques, in economètrie, Colloques Internationaux du Centre National de la Recherce Scientifique, 40, 41-47; English version: the role of securities in the optimal allocation of risk-bearing. Review of Economic Studies, 31, 91-96.

Aumann, R.J., 1961. The core of a cooperative game without side payments. Transactions of the American Mathematical Society 98, 539-552.

Debreu, G., 1972. Smooth preferences. Econometrica 40, 603-615.

Gale, D., 1978. The core of a monetary economy without trust. Journal of Economic Theory 19, 456-491.

Geanakoplos, J.D., Polemarchakis, H.M., 1986. Existence, regularity, and constrained suboptimality of competitive allocations when the asset market is incomplete. In: Heller, W.P., Starr, R.M., Starrett, D.A. (Eds.), Uncertainty, Information and Communication: Essays in Honor of K.J. Arrow, vol. III. Cambridge University Press, Cambridge, pp. 65-96.

Koutsougeras, L.C., 1998. A two-stage core With applications to asset market and differential information economies. Economic Theory 11, 563-584.

Kranich, L., Perea, A., Peters, H., 2001. Core Concepts for Dynamic TU-game, METEOR Discussion Paper 01/024, Universiteit Maastricht.

Predtetchinski, A., Herings, P.J.J., Perea, A., 2002. The Weak Sequential Core for Two-period Economies, METEOR Discussion Paper 02/011, Universiteit Maastricht, pp. 1-30.

Repullo, R., 1988. The core of an economy with transaction costs. Review of Economic Studies 55, 447-458.

Vohra, R., 1999. Incomplete information, incentive compatibility, and the core. Journal of Economic Theory 86, $123-147$. 